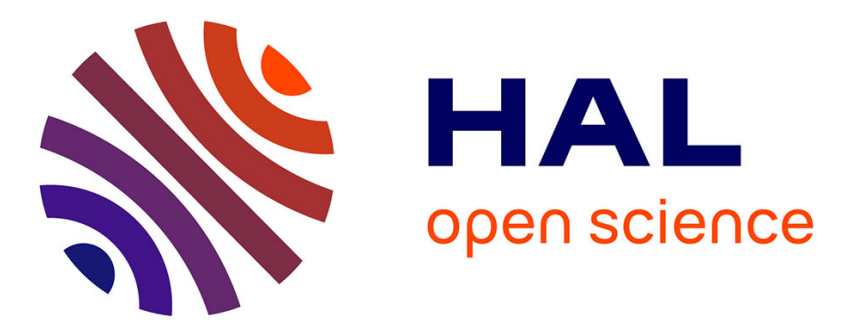

\title{
Synthesis, Encapsulation, and Performance Analysis of Large Deformation Tri-layer Polypyrrole Actuator
} Amélie Cot, Mohamed Taha Chikhaoui, Kanty Rabenorosoa, Patrick Rougeot, Nicolas Andreff

\section{- To cite this version:}

Amélie Cot, Mohamed Taha Chikhaoui, Kanty Rabenorosoa, Patrick Rougeot, Nicolas Andreff. Synthesis, Encapsulation, and Performance Analysis of Large Deformation Tri-layer Polypyrrole Actuator. International Conference on Advanced Intelligent Mechatronics, Jul 2017, Munich, Germany. hal02868210

\section{HAL Id: hal-02868210 https://hal.science/hal-02868210}

Submitted on 15 Jun 2020

HAL is a multi-disciplinary open access archive for the deposit and dissemination of scientific research documents, whether they are published or not. The documents may come from teaching and research institutions in France or abroad, or from public or private research centers.
L'archive ouverte pluridisciplinaire HAL, est destinée au dépôt et à la diffusion de documents scientifiques de niveau recherche, publiés ou non, émanant des établissements d'enseignement et de recherche français ou étrangers, des laboratoires publics ou privés. 


\title{
Synthesis, Encapsulation, and Performance Analysis of Large Deformation Tri-layer Polypyrrole Actuator
}

\author{
Amélie Cot, Mohamed Taha Chikhaoui*, Kanty Rabenorosoa*, Patrick Rougeot, Nicolas Andreff*, \\ *IEEE Member
}

\begin{abstract}
This paper reports the synthesis of an electroactive polymer actuator in polypyrrole (PPy) on a polyvinylidene difluoride (PVDF) substrate. The technological development is detailed. This study reports our investigation on a tri-layer PPy actuator for large deformations and comparison with literature modeling. We also investigate their use towards real applications by introducing lifetime measurement, encapsulation and closed loop control.
\end{abstract}

\section{INTRODUCTION}

The use of electroactive polymers (EAPs) as electromechanical transducers in different applications appears in recent years [1] especially for biomedical applications [2]. These polymers are able to respond to an electrical stimulation by a change of dimensions or shapes. Compared to existing technologies, they also have a number of advantages as flexibility, low weight, large deformations, possibility of miniaturization and low cost fabrication. Two types of EAPs are usually studied as actuators : (i) dielectrical EAPs (through electronic EAPs) which have interesting properties as good deformation $(10-150 \%)$ and high dynamic [3]. However, the necessity of high voltages to activate the system can limit their application and (ii) conducting polymers (CPs) (through ionic EAPs) which volume variations are induced by ion displacement between an electrolyte and the material. They also present interesting characteristics as transducers due to their direct conversion between electrical energy and mechanical energy. This kind of properties is augmented by their low operating voltage $(<3 \mathrm{~V})$, large deformation (up to 20\%), simple construction and biocompatibility [2]. Different CPs were investigated in the literature and two materials are often highlighted : polypyrrole (PPy) and poly(3,4-ethylenedioxythiophene) (PEDOT) [4], [5], [6]. The comparison of their performances are largely discussed [7], [8].

In our study, we choose to focus on one of the most known $\mathrm{CP}, \mathrm{PPy}$ which up to now is widely used for biomedical application as active catheters [9], artificial muscle [10] and micromanipulation [11]. This study reports our investigation on a tri-layer PPy actuator for large deformations where this state of the art is generally limited to small deformations [12], [13]. We also investigate their use towards real applications by introducing lifetime measurement, encapsulation and closed loop control. This paper is organized as follows. Section II describes in details the micro-actuators synthesis

All authors are with the FEMTO-ST Institute, AS2M department, Univ. Bourgogne Franche-Comté, UFC/CNRS/ENSMM, 24 rue Savary, F-25000 Besançon, France. and highlights the influent parameters. Electromechanical characterization of the synthetized micro-actuators is proposed in Section III. In Section IV, the state of the art model validation and limits are discussed and Section V presents the investigation toward the real use of PPy through encapsulation and life-time characterization. Closed loop control is proposed in Section VI in order to show precise control of non-linear actuator. Section VII concludes the paper and details fundamentals.

\section{MICROACTUATORS SYNTHESIS}

\section{A. Polypyrrole electrosynthesis}

Polypyrrole synthesis follows the protocol of Hara et al. [14], [10]. All samples were prepared by electrochemical process using a Voltatstat PGP 201 (TacusselRadiometerAnalytical SA, France) controlled by Voltamaster4 software. A standard three-electrodes system was linked to the potentiostat and composed by an $\mathrm{Ag} / \mathrm{AgCl}$ electrode as the reference electrode and a platinum grid as the counterelectrode (Figure 1). The working electrode was a PVDF membrane Immobilion P from Millipore (according to product specifications :hydrophobic, thickness $125 \mu \mathrm{m}$, pore size $0.45 \mu \mathrm{m}$, porosity $70 \%$ ).

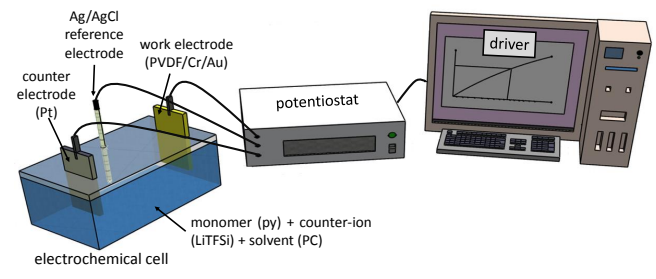

Fig. 1: Potentiostat with three electrodes system.

Before electrochemical process, the PVDF membrane was previously covered by sprayed chromium $(20 \mathrm{~nm})$ and gold $(40 \mathrm{~nm})$ to bring the conductivity. Several thicknesses of chromium and gold were experimented in order to obtain enough conductivity without introducing too much mechanical stresses [15]. Pyrrole was from Sigma Aldrich (99\% pure) and was distilled under reduced pressure before use $(0.1 \mathrm{M})$. Lithium bistrifluoromethanesulfonimidate (LiTFSi) was from Sigma Aldrich and used as electrolytic salt (0.06 M). At fist, cyclic voltammetry was processed on a gold substrate to make sure of the quality of the solution. 
All experiments were carried out in a freezer at $-20 \pm 2{ }^{\circ} \mathrm{C}$ galvanostatically during 10 hours. Current density was fixed at $0.2 \mathrm{~mA} / \mathrm{cm}^{2}$. Low temperature and low current density were chosen for the good mechanical properties [16] and improved actuation properties [17] of the synthesized trilayer actuator.

\section{ELECTROMECHANICAL CHARACTERIZATION}

\section{A. Principle}

CPs have the property to move from a state called "doped" to another state "undoped", reversibly, by a simple redox reaction. It induces an ions exchange with the electrolytic medium to conserve the electroneutrality of the system. These mechanisms result in an expansion or a contraction of volume of the polymer and hence to mechanical working. A trilayer actuator is composed of two polymer electrodes on each side of the substrate serving as an electrolyte reservoir and permeable to ions. The molecular mechanism will depend essentially on the nature and size of the ions involved and their state of solvation. In our case, LiTFSi dissociates when it is put in a solvent into a cation $\left(\mathrm{Li}^{+}\right.$,

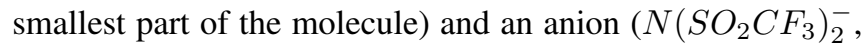
biggest part of the molecule) following equation 1 .

$$
\mathrm{LiN}\left(\mathrm{SO}_{2} \mathrm{CF}_{3}\right)_{2} \leftrightharpoons \mathrm{Li}^{+}+\mathrm{N}\left(\mathrm{SO}_{2} \mathrm{CF}_{3}\right)_{2}^{-}
$$

As shown in Figure 2, when a voltage is applied to the anode, PPy takes its oxidized form due to the gain of electron. Thus to compensate the gain of negative charges, cations $\mathrm{Li}^{+}$contained is the porous substrate are inserted in the polymer whereas anions $N\left(S_{2} \mathrm{CF}_{3}\right)_{2}^{-}$go out of the polymer. Consequently to the respective sizes of anions and cations, the reaction results in the contraction of the polymer. The opposite reaction occurs at the cathode resulting in an expansion of the polymer.

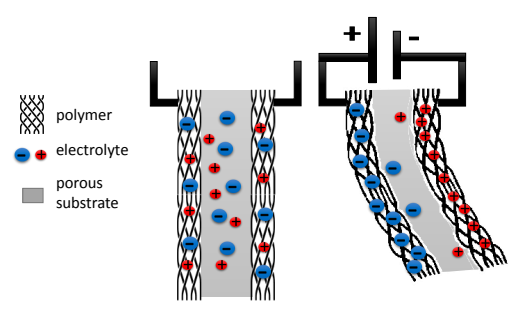

Fig. 2: General working principal of conducting polymer when the left electrode is oxidized and the right electrode is reduced.

Then there is induction of a mechanical movement by chemical reaction obtained by applying a voltage.

\section{B. Actuation setup}

To characterize the obtained mechanical movement, we have developed a test bench. First step is to define the actuator size, $2 \mathrm{~mm}$ wide and $30 \mathrm{~mm}$ long. Then it is necessary to soak the actuator with the actuating solution

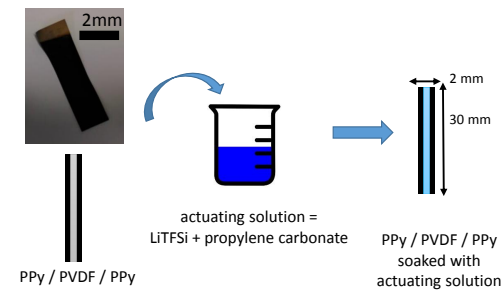

Fig. 3: Actuator preparation for tests

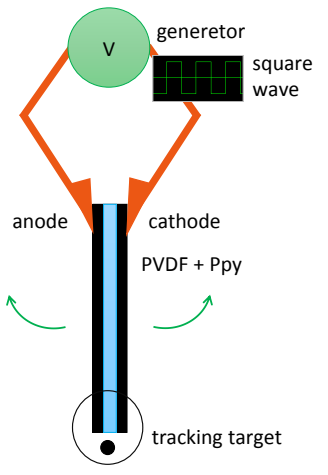

(a) Schema of the actuation set up.

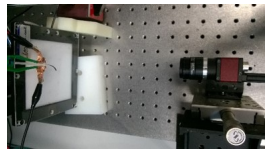

(b) Top view of the actuation setup.

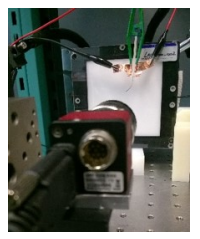

(c) Back view of the actuation setup.
Fig. 4: Actuation setup details

containing the electrolyte and the solvent (Figure 3).

Applied voltage and camera feedback are controlled by MATLAB Simulink programm using Visual Servoing Platform with a dedicated blockset CVlink. Camera (IEE 1394 Guppy Firewire) is placed in front of the actuation platform (Figures $4 \mathrm{~b}$ and $4 \mathrm{c}$ ) in a way to visualize the side of the actuator in its thickness. The actuator is held at one of its extremity by a small gripper made conductive with copper (Figure 4a). Voltage is provided via a National Instruments multifunction data acquisition module (USB-6211), which can manage between $\pm 10 \mathrm{~V}$ with a resolution of $3.512 \mathrm{mV}$. It also measures the resulting current via a $10 \Omega$ resistor. Position tracking is processed adding a physical marker at the free extremity of the actuator (Figure 4a). It is a circular piece of light paper (to avoid adding too much weight on the actuator) with a black point of nearly $1 \mathrm{~mm}$ diameter (enough to be clearly detected and tracked by the camera). An evaluation of the shape factor of the black point is done using its isoperimetric quotient after a first detection when the marker is set. Then a tresholding of image allows the program to precisely detect the tracking target.

\section{Characterization of PPy actuator}

1) Step response: The objective is to obtain the time response of the tri-layer actuator for different applied voltages (step response). The setup, previously described, is used to measure the deformation of the whole actuator. The offline image processing enables the angle detection according to 
the time as shown in Figure 5.

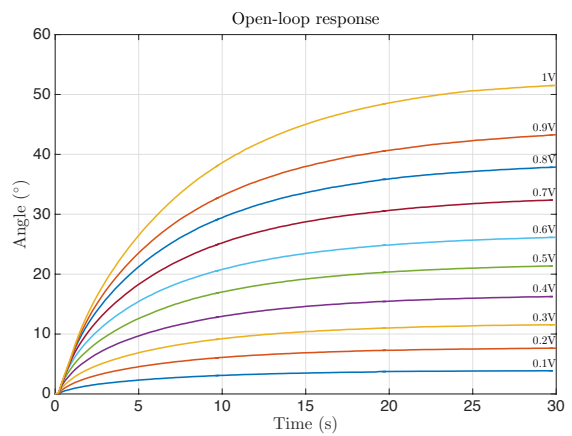

Fig. 5: Response time for different applied voltages.

It is observed that the angle depends on the applied voltage and the response time is difficult to measure due to the drift. The system is non linear and the response time is variable.

\begin{tabular}{|c|c|}
\hline Step amplitude $(V)$ & Transfert function \\
\hline 0.1 & $G(p)=\frac{38.1}{\left(1+1.10^{-6} p\right)(1+5.5 p)}$ \\
\hline 0.2 & $G(p)=\frac{38.6}{(1+0.9 p)(1+7.5 p)}$ \\
\hline 0.3 & $G(p)=\frac{39.1}{(1+0.91 p)(1+7.6 p)}$ \\
\hline 0.4 & $G(p)=\frac{4.3}{(1+1.3 p)(1+7.9 p)}$ \\
\hline 0.5 & $G(p)=\frac{41.9}{\left(1+1.10^{-6} p\right)(1+5.6 p)}$ \\
\hline 0.6 & $G(p)=\frac{44.2}{(1+1.2 p)(1+7.7 p)}$ \\
\hline 0.7 & not identified \\
\hline 0.8 & not identified \\
\hline 0.9 & not identified \\
\hline 1 & not identified \\
\hline
\end{tabular}

TABLE I: Identification results of the actuator transfert function.

Table I shows that the different transfert functions are identified for each applied voltage. From $0.7 \mathrm{~V}$ to $1 \mathrm{~V}$, the identification is not performed because the transient part is not finished and the best fit is less than $90 \%$.

2) Bode diagram: Different sinusoidal voltages are applied in order to perform the frequency response of the trilayer PPy actuator. According to the proposed setup, the maximum frequency is limited to $1 \mathrm{~Hz}$ due to the sampling time $(0.2 \mathrm{~s})$ of MATLAB/Simulink and the dynamic of the actuator. The result is shown in Figure 6.

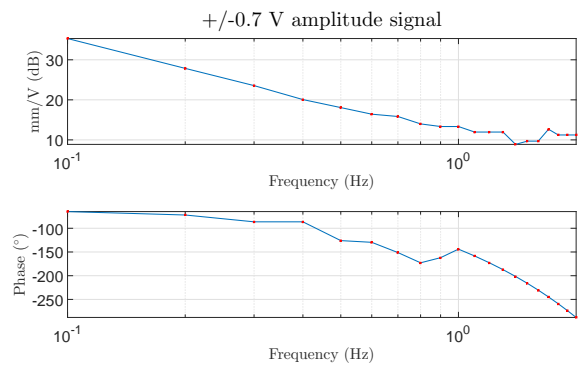

Fig. 6: Bode diagramm of the microactuator.

It is observed for $0.1 \mathrm{~Hz}$, the phase is already $-64.8^{\circ}$ and it can reach $-288^{\circ}$ for $2 \mathrm{~Hz}$.
3) Curvature measurements: The movement is strongly dependent of the applied voltage. So to avoid perturbations during measurements and hysteresis phenomenon, a neutralization procedure is done between each voltage step. So the actuator is returned to its original position before a new voltage is applied. As shown is Figure 7, the actuator has a prebending induced by mechanical stresses caused by the several steps of polymer synthesis. Nevertheless, it is clear that more the voltage value is far from the original value of $0 \mathrm{~V}$, more the actuator has a large range of motion and greater curvature. Until a potential of $0.4 \mathrm{~V}$, the actuator has a quite linear behaviour. From a votage value of $0.5 \mathrm{~V}$, the actuator takes a very tight bending radius (see table II). The movement of the actuator becomes very difficult to measure from $0.7 \mathrm{~V}$ because the free end reaches a higher elevation above pinned point at the actuator base.

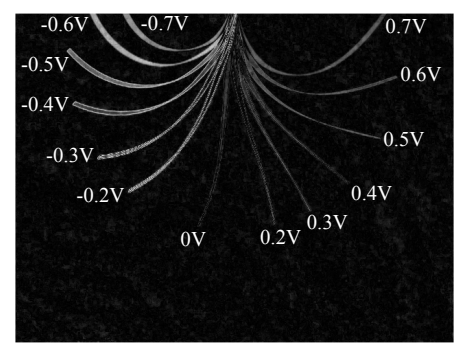

Fig. 7: Actuation results through superposition of different images obtained during the test.

\begin{tabular}{|c|c|c|c|}
\hline \multicolumn{2}{|c|}{$\begin{array}{c}\text { Applied } \\
\text { voltage }(V)\end{array}$} & $\begin{array}{c}\text { Maximum } \\
\text { curvature }\left(\mathrm{m}^{-1}\right)\end{array}$ & \multicolumn{2}{|c|}{$\begin{array}{c}\text { Bending } \\
\text { radius }(\mathrm{m})\end{array}$} \\
\hline-0.2 & 0.2 & $24 \mid 12$ & $4.10^{-2} \mid 8.10^{-2}$ \\
\hline-0.3 & 0.3 & $34 \mid 20$ & $3.10^{-2} \mid 5.10^{-2}$ \\
\hline-0.4 & 0.4 & $48 \mid 30$ & $2.10^{-2} \mid 3.3 .10^{-2}$ \\
\hline-0.5 & 0.5 & $61 \mid 42$ & $1.6 .10^{-2} \mid 2.3 .10^{-2}$ \\
\hline-0.6 & 0.6 & $78 \mid 60$ & $1.3 .10^{-2} \mid 1.6 .10^{-2}$ \\
\hline-0.7 & 0.7 & $100 \mid 78$ & $1.10^{-2} \mid 1.3 .10^{-2}$ \\
\hline
\end{tabular}

TABLE II: Maximum curvatures obtained for several positive and negative voltages.

\section{MODELING VALIDATION}

\section{A. Analytical modeling}

Several modeling of tri-layer actuators have already been proposed, linear [18], [19], [20] or non linear [21], [13], [22]. We choose to focus on Alici's modeling [23] because it is on conditions as close to ours : same conducting polymer, same substrate, electrolytes of similar size, comparable actuators dimensions. Moreover Alici's modeling takes into account most of the properties of the actuator. Indeed it considers electrical properties as the link beetween the applied voltage and the obtained oxidation state. It also considers chemical parameters as the rate of ions diffusion or molecular weights. And finally, it introduced mechanical properties including elastic modulus and real dimensions of each component 
of the actuator (polymer and substrate). Alici's modeling enables to describe bending curves of the actuator for several voltages. It is based on the understanding of the force output at the actuator's tip and the induced bending moments to obtain a bending curve model via Hooke's law :

$$
v^{\prime \prime}-\left[1+\left(v^{\prime}\right)^{2}\right] \frac{\left[\alpha V C / b L\left(h_{2}-h_{1}\right)\right] b\left(h_{2}^{2}-h_{1}^{2}\right)}{E_{P P y}\left(2 b\left(h_{2}^{3}-h_{1}^{3}\right) / 3\right)+E_{P V D F}\left(2 b h_{1}^{3} / 3\right)}
$$

which can be simplified :

$$
v^{\prime \prime}-\left[1+\left(v^{\prime}\right)^{2}\right] \frac{3 \alpha V C\left(h_{2}+h_{1}\right)}{2 b L\left[E_{P P y}\left(h_{2}^{3}-h_{1}^{3}\right)+E_{P V D F} h_{1}^{3}\right.}=0
$$

To compare modeling with experimental results, it is necessary to resolve this nonlinear second-order differential equation describing the bending curves (equation 2). Then it has been solved numerically, integrating our real experimental parameters. Parameters of the equation 3 are given in table III.

\begin{tabular}{|c|c|c|}
\hline Name & Variable & Value \\
\hline$v$ & verticalposition & $/$ \\
\hline$\alpha$ & corrective factor & to determine \\
\hline$V$ & voltage & from 0.2 to $0.7 \mathrm{~V}$ \\
\hline$C$ & capacitance & to determine \\
\hline$h_{1}$ & PVDF half - thickness & $55 \mu \mathrm{m}$ \\
\hline$h_{2}$ & PVDFhalf - thickness & $55 \mu \mathrm{m}+10 \mu \mathrm{m}$ \\
& + PPythickness & $=65 \mu \mathrm{m}$ \\
\hline$b$ & actuator widht & $3 \mathrm{~mm}$ \\
\hline$L$ & actuator lenght & $20 \mathrm{~mm}$ \\
\hline$E_{P P y}$ & $P$ Py Young ${ }^{\prime}$ modulus & $80 \mathrm{MPa}[24]$ \\
\hline$E_{P V D F}$ & $P V D F$ Young ${ }^{\prime}$ s modulus & $440 \mathrm{MPa}[24]$ \\
\hline
\end{tabular}

TABLE III: Model parameters.

To complete parameters, it is necessary to characterize experimentally the capacitance of the actuator. Indeed, as the mechanical behavior of conducting polymer is linear with exchanged, we need to define a relation between the applied voltage and the exchange of charges. A chronoamperometry with the potentiostat and the three electrode system (Figure 1) is performed in the actuation solution in order to obtain the exchange of charges between the solution and the PPy (Figure 8). Then the equation 4 is applied :

$$
\Delta Q=\Delta V C
$$

where $\Delta Q$ is the exchange of charge, $\Delta V$ is the applied voltage and $C$ is the capacitance. A simple integration of the current data enables to calculate the capacitance. For the considered actuator, the value of $C$ is $0.9 .10^{3} \mathrm{~S}$.

The latest parameter $\alpha$ is the corrective factor which permits to link the volume of the conductive polymer and the exchange of charge. It will be defined to obtain the best-fit between modeling and experimental data. It is not absolutely constant but stays in an interval between $0.94 .10^{-1}$ and $1.125 .10^{-1}\left(\mathrm{~F} / \mathrm{m}^{2}\right) /\left(\mathrm{C} / \mathrm{m}^{3}\right)$.

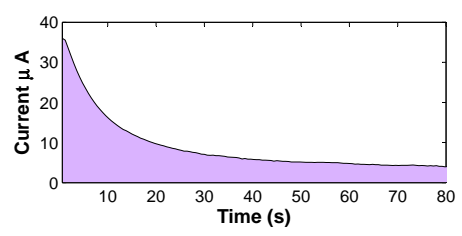

Fig. 8: Experimental current response of tri-layer actuator under a voltage step input.

\section{B. Comparison of modeling and experimental results}

Comparison between experimental data and modeling results are presented in Figure 9. Blue lines represent modeling results and colored points symbolize experimental data for several voltages between $0.2 \mathrm{~V}$ and $0.7 \mathrm{~V}$. Bending curves are totally similar for the modeling and experimental points until a voltage of $0.4 \mathrm{~V}$. When the applied voltage is higher than $0.4 \mathrm{~V}$, the model is only valid for a limited part of the curve. The model is no longer valid because the actuator is too long and that the model used may not describe the curves for $0.6 \mathrm{~V}$ and $0.7 \mathrm{~V}$, because the bending angle exceeds 90 . Mechanical response could be greater for long actuator than for smaller ones. Furthermore, the developed actuators have a thinner thickness of PPy inducing less mechanical prestress.

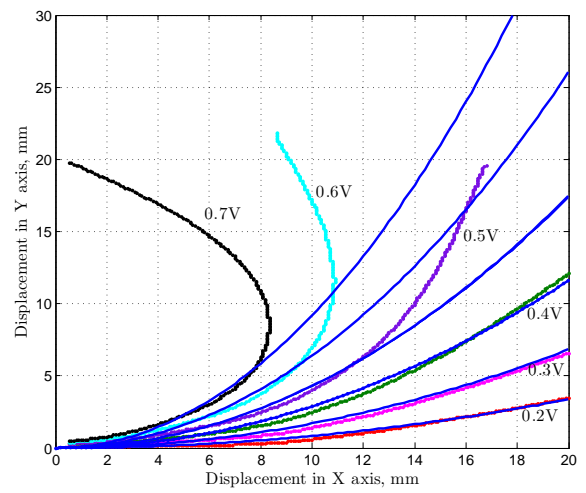

Fig. 9: Comparison of experimental (colored points) and modeling results (blue lines).

\section{ENCAPSULATION}

One of the problems limiting the duration of the $\mathrm{CP}$ is evaporation of the actuating solution stored in the porous PVDF. Several solutions can be envisaged to prevent this, such as the use of solid electrolytes, but they have low ionic conductivity [25]. Some encapsulation methods of the actuating liquid by specific polymers (SIBS) show good results but are difficult to achieve [26]. For this reason, we choose to use a well known polymer to process encapsulation, polydimethylsiloxane (PDMS). PDMS is the most widely used silicon-based organic polymer, and is particularly known for its unusual rheological properties. Further, it is non-toxic and inert, that is important for biomedical applications [27]. 


\section{A. Experimental encapsulation}

PDMS was prepared with a commercial product MED24220 Silicone technology from NUSIL. It is composed by two pumps, one containing the monomer, the other containing the cross-linking. After mixing the 2 parts, the air bubbles are removed using a vacuum pump. Then, a thin layer of PDMS is spread on both sides of the actuator. The encapsulation of the actuator was made just after immersed it in the actuating solution to charge the maximum electrolyte. Then an annealing at $70^{\circ} \mathrm{C}$ is performed during one hour. The resulting encapsulation seems to be uniform (Figure 10b), the nodular structure of PPy layer (Figure 10a) is no longer visible.

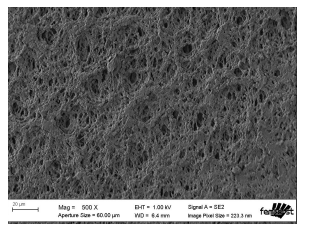

(a) MEB picture of PPy.

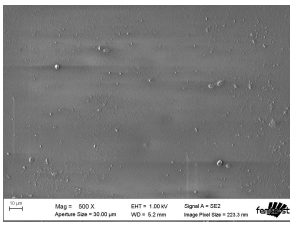

(b) MEB picture of PPy encapsulated by PDMS
Fig. 10: MEB pictures of non encapsulated and encapsulated samples.

\section{B. Results}

For lifetime measurements, two mediums have been experimented, air and water.

1) In air: Lifetime measurements in air are performed in order to determine if the evaporation of the actuating solution could be limited and if it brought an interest in the possible duration of use of the actuator. A lifetime measurement have been performed on a non encapsulated actuator and an encapsulated actuator during 4 hours. Results are shown is Figure 11. The first thing is that encapsulation reduces the mobility of the actuator providing more mechanical prestress. Indeed the displacement of the actuator is divided by a factor 3 when it is encapsulated. So, for a four hours actuating, the loss of mobility is $60 \%$ for the non encapsulated actuator and also for the encapsulated one. Nevertheless, the tiredness of the actuator is linear when it is encapsulated whereas the the displacement drop is more brutal when it is not, so the behavior of the encapsulated actuator could be more easily predicted than the nude one.

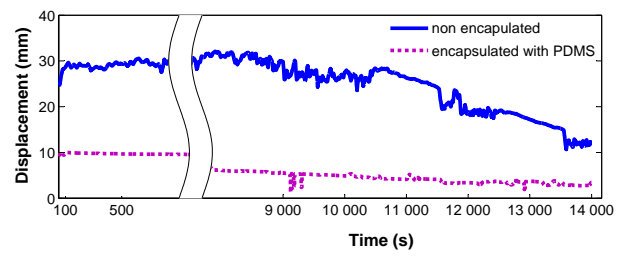

Fig. 11: Lifetime measurements without encapsulation and with PDMS encapsulation in air.
2) In liquid medium: It is more difficult to characterize the actuation in water because the diffraction of the medium makes it impossible tracking the actuator. Nevertheless, video results shows that the lifetime of the encapsulated actuator (24 min) is more than two times longer compared to the lifetime of the non-encapsulated one $(10 \mathrm{~min})$. Indeed, the ionic diffusion of the electrolyte in water is quicker when the polymer is nude.

\section{CLOSED-LOOP CONTROL}

The detected position is expressed in terms of angular measure considering the clamping point at the gripper. In order to properly control the end-effector position, a closedloop scheme based on visual feedback is proposed. Following the angular measure previously detailed, the experiments were led with respect to the block diagram in Figure 12. A PID controller is used to compensate for the measured error between the desired angle $\alpha_{d}$ and the measured one $\alpha$. Several square inputs of different amplitudes were tested as well as ramp signals as depicted in Figure 13. In order to protect the micro-actuator from damage, a voltage limitation of $2.5 \mathrm{~V}$ was set.

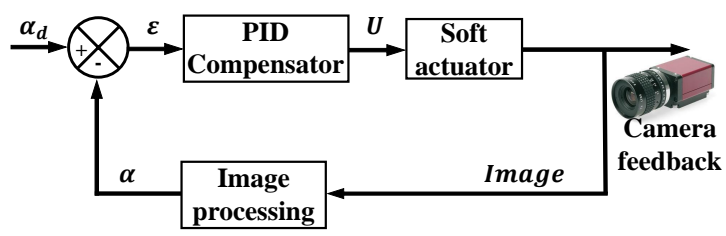

Fig. 12: Block diagram for the visual servoing based curvature control.

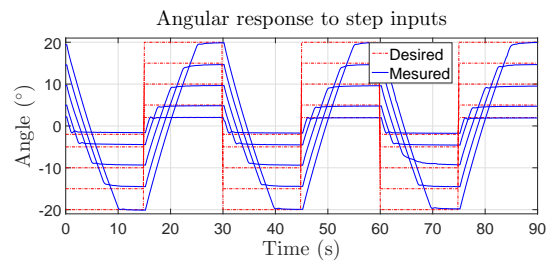

(a) Responses to square inputs.

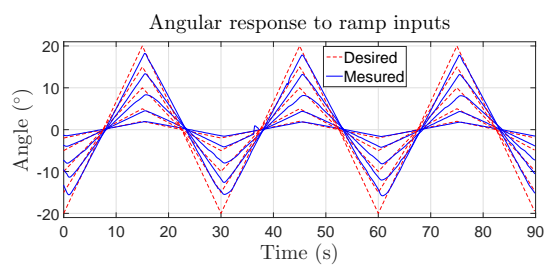

(b) Responses to sawtooth inputs.

Fig. 13: End effector angular response with square and sawtooth control inputs.

The tested angular inputs range from 2 to $20^{\circ}$. The response analysis show a satisfactory steady state error underneath $5 \%$ (Figure 13a) and a tracking error of $10 \%$ (Figure 13b) due to the actuator dynamics. These promising results open the scope for robotic applications that require 
high position accuracy. The average response time, of few seconds here, can be substantially reduced with the suitable gain in the compensator while risking to alter the actuator life cycle with high voltage inputs.

\section{CONCLUSION AND FUTURE WORKS}

A tri-layer actuator was proposed and its complete synthesis and characterization were performed and reported in this paper. Actuation test shown very large deformations with bending radius of $1.10^{-2} \mathrm{~m}$ for low voltages, under $1 \mathrm{~V}$ with a good repeatability. A close modeling from the literature was adapted to fit with parameters of our tri-layer actuator. We have shown that modeling is validated for low voltages (less than $0.4 \mathrm{~V}$ ) for large deformations but not above $0.5 \mathrm{~V}$ for the considered actuator dimension.. Then a easy encapsulation solution is proposed to enhance the lifetime in the water and linearise the actuator behavior in air according to the time. In addition, a closed loop control have been proposed and shown very promising results concerning the position control perfromances (steady state error 5\% and tracking error 10\%). Future works will be concentrated on the transfer of the actuating results on PVDF planar substrates towards flexible tubes with electrodes on each side for actuation in two degrees of freedom. Moreover, the assembly of PPy with PEDOT is considered to enhance the response time of the actuator without altering large deformation movements.

\section{ACKNOWLEDGMENT}

This work has been supported by the Labex ACTION project (contract "ANR-11-LABX-0001-01"), and by NEMRO project (contract ANR-14-CE17-0013), by the Equipex ROBOTEX project (contract "ANR-10-EQPX-44-01"), by the French RENATECH network and its FEMTO-ST technological facility.

\section{REFERENCES}

[1] G. Alici, B. Mui, and C. Cook, "Bending modeling and its experimental verification for conducting polymer actuators dedicated to manipulation applications," Sensors and Actuators A : Physical, vol. 126, no. 2, pp. 396-404, 2006.

[2] F. Carpi, E. Smela et al., "Biomedical applications of electroactive polymer actuators," 2009.

[3] R. Pelrine, R. Kornbluh, Q. Pei, and J. Joseph, "High-speed electrically actuated elastomers with strain greater than 100\%," Science, vol. 287, no. 5454, pp. 836-839, 2000.

[4] U. L. Zainudeen, M. A. Careem, and S. Skaarup, "Pedot and ppy conducting polymer bilayer and trilayer actuators," Sensors and Actuators B : Chemical, vol. 134, no. 2, pp. 467-470, 2008.

[5] C. Plesse, F. Vidal, D. Teyssié, and C. Chevrot, "Conducting polymer artificial muscle fibres : toward an open air linear actuation," Chemical Communications, vol. 46, no. 17, pp. 2910-2912, 2010.

[6] A. Maziz, "Microactionneurs à base de polymères conducteurs électroniques : Vers 1 intégration aux microsystèmes par de nouveaux procédés d élaboration," Ph.D. dissertation, Cergy-Pontoise, 2014.

[7] R. Temmer, A. Maziz, C. Plesse, A. Aabloo, F. Vidal, and T. Tamm, "In search of better electroactive polymer actuator materials : Ppy versus pedot versus pedot-ppy composites," Smart Materials and Structures, vol. 22, no. 10, p. 104006, 2013.

[8] W. Lu, A. G. Fadeev, B. Qi, E. Smela, B. R. Mattes, J. Ding, G. M. Spinks, J. Mazurkiewicz, D. Zhou, G. G. Wallace et al., "Use of ionic liquids for $\pi$-conjugated polymer electrochemical devices," Science, vol. 297, no. 5583, pp. 983-987, 2002.
[9] T. Shoa, N. R. Munce, V. Yang, and J. D. Madden, "Conducting polymer actuator driven catheter : overview and applications," in SPIE Smart Structures and Materials + Nondestructive Evaluation and Health Monitoring. International Society for Optics and Photonics, 2009, pp. 72 871J-72 871J.

[10] S. Hara, T. Zama, W. Takashima, and K. Kaneto, "Artificial muscles based on polypyrrole actuators with large strain and stress induced electrically," Polymer journal, vol. 36, no. 2, pp. 151-161, 2004.

[11] G. Alici and N. N. Huynh, "Performance quantification of conducting polymer actuators for real applications : a microgripping system," Mechatronics, IEEE/ASME Transactions on, vol. 12, no. 1, pp. 73-84, 2007.

[12] S. John and G. Alici, "Towards micro and nano manipulation systems : behaviour of a laminated polypyrrole (ppy) actuator driving a rigid link," Faculty of Engineering-Papers, p. 67, 2005.

[13] G. Alici, "An effective modelling approach to estimate nonlinear bending behaviour of cantilever type conducting polymer actuators," Sensors and Actuators B : Chemical, vol. 141, no. 1, pp. 284-292, 2009.

[14] S. Hara, T. Zama, S. Sewa, W. Takashima, and K. Kaneto, "Highly stretchable and powerful polypyrrole linear actuators," Chemistry Letters, vol. 32, no. 7, pp. 576-577, 2003.

[15] L. Barrand and N. Andreff., "Towards polypyrrole actuated flexible endomicroscope : Synthesis," in JNTE, 2015.

[16] E. J. Oh, K. S. Jang, S. Y. Park, S. S. Han, and J. S. Suh, "Electrochemical synthesis and characterization of strechable polypyrrole films," Molecular Crystals and Liquid Crystals Science and Technology. Section A. Molecular Crystals and Liquid Crystals, vol. 371, no. 1, pp. 243-246, 2001.

[17] S. Maw, E. Smela, K. Yoshida, P. Sommer-Larsen, and R. B. Stein, "The effects of varying deposition current density on bending behaviour in ppy (dbs)-actuated bending beams," Sensors and Actuators A : Physical, vol. 89, no. 3, pp. 175-184, 2001.

[18] J. D. Madden, P. G. Madden, and I. W. Hunter, "Polypyrrole actuators : modeling and performance," in SPIE's 8th Annual International Symposium on Smart Structures and Materials. International Society for Optics and Photonics, 2001, pp. 72-83.

[19] I. Must, M. Anton, M. Kruusmaa, and A. Aabloo, "Linear modeling of elongated bending eap actuator at large deformations," in SPIE Smart Structures and Materials+ Nondestructive Evaluation and Health Monitoring. International Society for Optics and Photonics, 2009, pp. 728 723-728 723 .

[20] A. Della Santa, D. De Rossi, and A. Mazzoldi, "Characterization and modelling of a conducting polymer muscle-like linear actuator," Smart Materials and Structures, vol. 6, no. 1, p. 23, 1997.

[21] M. Farajollahi, J. D. Madden, and F. Sassani, "Non-linear time variant model intended for polypyrrole-based actuators," in SPIE Smart Structures and Materials + Nondestructive Evaluation and Health Monitoring. International Society for Optics and Photonics, 2014, pp. 90561T-90561T.

[22] Y. Fang, T. J. Pence, and X. Tan, "Nonlinear elastic modeling of differential expansion in trilayer conjugated polymer actuators," Smart Materials and Structures, vol. 17, no. 6, p. 065020, 2008.

[23] G. Alici, B. Mui, and C. Cook, "Bending modeling and its experimental verification for conducting polymer actuators dedicated to manipulation applications," Sensors and Actuators A : Physical, vol. 126, no. 2, pp. 396-404, 2006.

[24] G. M. Spinks, L. Liu, G. G. Wallace, and D. Zhou, "Strain response from polypyrrole actuators under load," Advanced Functional Materials, vol. 12, no. 6-7, pp. 437-440, 2002.

[25] J. D. Madden, R. A. Cush, T. S. Kanigan, C. J. Brenan, and I. W. Hunter, "Encapsulated polypyrrole actuators," Synthetic Metals, vol. 105, no. 1, pp. 61-64, 1999.

[26] S. Naficy, N. Stoboi, P. G. Whitten, G. M. Spinks, and G. G. Wallace, "Evaluation of encapsulating coatings on the performance of polypyrrole actuators," Smart Materials and Structures, vol. 22, no. 7, p. $075005,2013$.

[27] A. Mata, A. J. Fleischman, and S. Roy, "Characterization of polydimethylsiloxane (pdms) properties for biomedical micro/nanosystems," Biomedical microdevices, vol. 7, no. 4, pp. 281-293, 2005. 